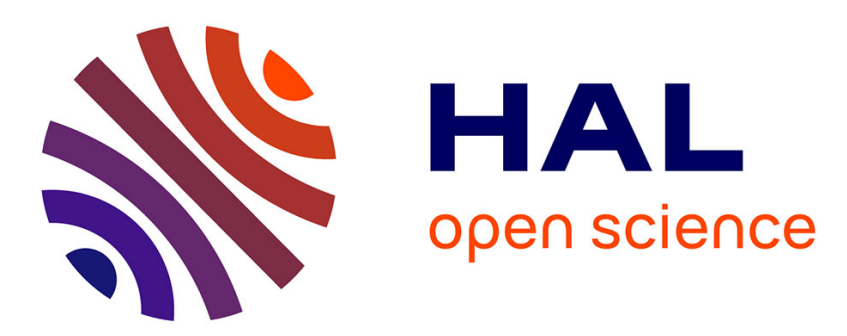

\title{
La mesure comptable des marques à l'heure de l'intégration européenne: une disparité préoccupante Élisabeth Walliser
}

\section{To cite this version:}

Élisabeth Walliser. La mesure comptable des marques à l'heure de l'intégration européenne: une disparité préoccupante. 20ÈME CONGRES DE L'AFC, May 1999, France. pp.CD-Rom. halshs00587776

\section{HAL Id: halshs-00587776 \\ https://shs.hal.science/halshs-00587776}

Submitted on 21 Apr 2011

HAL is a multi-disciplinary open access archive for the deposit and dissemination of scientific research documents, whether they are published or not. The documents may come from teaching and research institutions in France or abroad, or from public or private research centers.
L'archive ouverte pluridisciplinaire HAL, est destinée au dépôt et à la diffusion de documents scientifiques de niveau recherche, publiés ou non, émanant des établissements d'enseignement et de recherche français ou étrangers, des laboratoires publics ou privés. 


\title{
$22^{\text {nd }}$ annual congress of the European Accounting Association 20ème congrès annuel de l'Association Française de Comptabilité
}

Bordeaux, 5-7 mai 1999

\author{
La mesure comptable des marques \\ à l'heure de l'intégration européenne : \\ une disparité préoccupante
}

\author{
Elisabeth WALLISER \\ Conservatoire National des Arts et Métiers \\ Chaire de Comptabilité et de Contrôle de Gestion \\ 292, rue St Martin \\ 75141 Paris cedex 03 \\ 0143412543 \\ e-mail : walliser@cnam.fr
}

$\mathrm{Au}$ moment où l'intégration européenne entre dans une phase décisive, notamment au plan financier (avènement de l'euro, coordination des bourses de valeurs...), on observe une diversité préoccupante des méthodes relatives à la mesure comptable des actifs incorporels, et plus particulièrement des marques.

Cette disparité s'observe à deux niveaux:

En premier lieu, au niveau des textes en vigueur dans les pays concernés. Une analyse comparative du dispositif institutionnel dans les principaux pays européens (France, Allemagne, Royaume-Uni) met en relief d'importantes différences doctrinales en matière de reconnaissance comptable des marques; différences accrues par l'hétérogénéité des méthodes d'évaluation.

En second lieu, au niveau des pratiques des entreprises. Une étude statistique, portant sur 150 sociétés réparties sur les trois pays étudiés et sur cinq secteurs d'activités, complétée par une enquête qualitative auprès de 22 responsables, permet de tester les différentes hypothèses issues du cadre institutionnel.

Il apparaît que le choix d'une méthode d'évaluation, bien que délicat, n'est pas un obstacle majeur à la reconnaissance des marques distinctement du goodwill. En revanche, le fait que, dans l'ensemble, l'effet pays l'emporte sur l'effet secteur, légitime le souci de rapprocher les cadres institutionnels et les pratiques des acteurs dans l'Europe de demain. 


\section{La mesure comptable des marques \\ à l'heure de l'intégration européenne : \\ une disparité préoccupante}

\section{Introduction}

Cette communication s'inscrit dans le cadre de la réflexion comptable sur les actifs immatériels. Les marques constituent des investissements immatériels stratégiques en tant qu'instrument de concurrence susceptible de capter et de fidéliser une partie de la demande (KAPFERER, 1996). L'importance des marques et plus largement des investissements immatériels n'est plus à démontrer. Cette conclusion a été émise par des groupes de travail, en France notamment dès le début des années 80 (CGP, 1982) ${ }^{1}$. Ces études n'ont pas pour autant résolu le problème lié à leur mesure à leur prise en compte à l'actif du bilan ${ }^{2}$ ni à leur évaluation.

La présente recherche part du constat qu'il existe un décalage entre l'importance des marques dans les actifs de l'entreprise et la faiblesse de leur mesure comptable; cette dernière étant définie comme l'ensemble des opérations en amont de la chaîne de traitement comptable de la marque : de l'identification de la marque en tant qu'actif à l'explicitation des méthodes employées pour son évaluation. La mesure comptable des marques ne peut pas être simplement appréhendée à travers le droit de propriété qui la matérialise. Nous nous intéressons en fait à l'ensemble des éléments liés à la marque qui apportent quelque chose à l'entreprise parce qu'ils donnent une plus-value aux produits et aux services : c'est le terme «capital-marque » (AAKER, 1994 ; BARWISE, 1993) qui est ainsi défini. Sa valeur provient de la position que l'entreprise avec marque détient sur le marché, par rapport à celle qui aurait résulté sans la marque et qui s'explique par le fait que la marque s'inscrit dans une logique de différenciation de l'offre.

A présent que les investissements immatériels ont acquis une reconnaissance économique il leur reste à acquérir une reconnaissance comptable. Nous nous situons donc dans une deuxième vague de travaux sur l'immatériel qui privilégie cette optique (CNC 1992, 1997 ; ASB 
1993, 1997 ; IASC 1995, 1998 ; HOARAU \& TERNISIEN, 1997)³. Derrière la prise en compte des marques c'est la pertinence du système comptable et sa capacité à représenter la réalité économique et financière de l'entreprise qui est posée. Traditionnellement tournée vers le passé dans un contexte où l'essentiel du patrimoine de l'entreprise était matériel, la comptabilité doit s'adapter à des éléments immatériels comme les marques dont une des caractéristiques principales est d'être en mesure de produire des richesses pour l'avenir.

Le manque de précisions, voire le flou, en matière de mesure comptable des investissements immatériels et des marques en particulier, peut avoir des conséquences importantes en matière de qualité de l'information émises par les entreprises à travers la publication de leurs comptes pour les entreprises au sein d'un même pays mais aussi sur un plan international, entre les pays du fait de l'opportunité laissée aux dirigeants de développer une politique comptable (CASTA, 1997). Nous nous inscrivons dans une optique d'harmonisation des normes institutionnelles et des pratiques. Notre démarche est donc essentiellement normative (COLASSE, 1996). Pour pouvoir réduire les différences, il faut tout d'abord pouvoir les expliquer. La question qui est posée est : Peut-on expliquer le décalage entre l'importance des marques et la faiblesse de leur mesure comptable à travers la difficulté de ladite mesure ?

Le programme de recherche a été défini à partir de trois orientations majeures

- le couplage «procédures de comptabilisation - méthodes d'évaluation» comme composantes essentielles de la mesure comptable. La prise en compte des marques à l'actif du bilan passe par une reconnaissance de celles-ci en tant qu'actif de l'entreprise et est conditionnée par la possibilité de disposer d'une méthode d'évaluation suffisamment fiable et pertinente pour satisfaire aux qualités de l'information comptable. La reconnaissance d'une marque ne peut se réduire à une condition d'évaluation sans l'expliciter. La mesure comptable des marques doit alors passer par une explicitation des méthodes proposées et employées pour juger de leur adaptation par rapport à l'objectif d'activation de la marque. 
- les niveaux institutionnels et comportementaux comme champs d'analyse complémentaires. Etant donné les débats de doctrine entourant la mesure comptable des marques, une étude au niveau institutionnel se révèle indispensable pour expliquer les réticences et le retard en la matière. L'analyse des pratiques ne peut alors qu'être complémentaire pour juger d'une éventuelle déconnexion de la pratique vis à vis de la doctrine ou au contraire d'une réelle congruence. Elle se justifie par les pratiques innovantes de certaines entreprises qui ont été révélatrices de ce débat sur la mesure comptable des marques. L'analyse conjointe des niveaux institutionnels et des comportements permettra d'apprécier si la position doctrinale se reflète dans les pratiques pour un même pays et, plus généralement, si la diversité constatée à un niveau institutionnel se retrouve dans les pratiques des entreprises.

- une comparaison internationale intervient au niveau de chacun des points précédents. Elle prend comme référence trois pays européens majeurs: la France, l'Allemagne et le Royaume- Uni. Le choix de la France et du Royaume Uni est guidé par le rôle actif que ces pays jouent en soumettant des réflexions ou des normes sur les investissements immatériels et parfois sur la marque. Par ailleurs, alors que l'on oppose généralement d'un point de vue comptable le Royaume-Uni d'un côté à la France et à l'Allemagne de l'autre, la prise en compte de l'Allemagne permettra de savoir si cette différence est vérifiée dans le cadre de la mesure comptable des marques.

\section{Des disparités au niveau du cadre institutionnel : des solutions contradictoires entre les pays}

\subsection{Le cadre non stabilisé de la mesure comptable des marques}

L'examen de textes doctrinaux a permis de juger des positions des différents pays en matière de reconnaissance des marques à l'actif et de confronter celles-ci à la récente norme internationale sur les investissements immatériels. C'est le mode d'acquisition qui conditionne 
encore la reconnaissance des marques. En ce qui concerne les marques acquises de manière isolée les trois pays n'avancent pas d'objection particulière quant à leur prise en compte à l'actif du bilan, tandis que le problème se complique dans le cas de la marque acquise lors d'un regroupement et a fortiori dans le cas de la marque développée de manière interne.

Le débat, initié par le Royaume-Uni ${ }^{4}$, vient de se conclure par une norme (ASB 1997). Il a gagné progressivement la France (CNC 1992, 1997) alors qu'en Allemagne aucune question similaire n'a, à ce jour, gagné la profession comptable ${ }^{5}$. Enfin, au niveau international, une norme sur les actifs immatériels (IASC 1998) est parue très récemment après avoir accumulé un retard de près de trois ans, précisément à cause du problème des marques. Les solutions provisoires ou définitives semblent, d'ores et déjà, contradictoires entre les différents pays.

La non-reconnaissance des marques créées est explicite en Allemagne (Code de Commerce) tandis que pour le Royaume-Uni et pour l'IASC, l'interdiction de reconnaître les marques développées en interne ne survient qu'après de nombreux débats permis par les propositions des « exposés-sondages » et leurs réponses. Sur ce point, il faut souligner la position novatrice de la France puisqu'elle est la seule à proposer une solution -qui ne vaut toutefois pas avis- au problème des marques créées et à se montrer en faveur de leur activation (CNC 1992). Ses propositions s'appuient sur le concept de «projet», qui avait déjà été utilisé pour la reconnaissance des frais de $R \& D$ et des logiciels, et conduisent à admettre l'activation des dépenses relatives aux marques lorsque certaines conditions très strictes sont remplies. Ses conclusions divergent ainsi de celles de l'IASC qui se fonde pourtant sur le même concept, puisque l'organisme international en fait un argument supplémentaire pour ne pas les reconnaître (IASC, 1997 E60\$44) 6 .

Rappelons cependant qu'il y a, dans les trois pays, toujours moyen de reconnaitre ces marques créées de manière indirecte lorsqu'elles ont été acquises lors d'un regroupement en leur affectant une part de l'écart de première consolidation. Toutefois, la plupart des textes s'orientent 
ici vers des conditions évitant l'arbitrage entre les actifs immatériels : c'est le cas des marques et du goodwill qui est bien souvent visé explicitement. Ainsi, l'ASB et l'IASC indiquent que l'affectation de l'écart de première consolidation aux marques acquises lors de regroupement n'est possible qu'à condition d'aligner le traitement du goodwill sur celui des marques ${ }^{7}$. En France, l'affectation de l'écart de première consolidation aux marques est fortement recommandée $(\mathrm{CNC}, 1990)$ tandis que la question de l'arbitrage n'a pas, pour l'instant, été abordée explicitement. Rappelons tout de même, que la doctrine française ne prévoit pas de durée maximum pour l'amortissement du goodwill ni d'amortissement pour la marque, ce qui permet un traitement similaire entre ces deux types d'actifs.

En ce qui concerne la politique privilégiée en matière de dépréciation de la marque, on aboutit parfois à des conclusions identiques, alors que les données de départ sont très différentes ou inversement à des conclusions différentes avec les mêmes données. Ainsi, l'Allemagne et l'IASC ont le même point de vue en ce qui concerne l'amortissement des marques sur une durée de vie limitée, ce qui peut paraître contradictoire étant donné la vision différente qu'ils ont de l'objectif des états financiers. En Allemagne, l'amortissement des marques et du goodwill est prévu sur des durées très courtes (HAMMAN, VON DER GATHEN, 1994), ce qui fait de ce pays une exception par rapport aux autres doctrines comptables étudiées. A l'inverse, alors que l'ASB et l'IASC ont tous deux instauré un « test de dépréciation » de la valeur de la marque, les conclusions des deux organismes diffèrent, puisque le premier permet d'opter pour une dépréciation par le biais de provisions pour certaines marques dont la durée de vie est considérée comme illimitée, tandis que le second maintient son obligation d'amortir les marques dans tous les cas.

Une conclusion commune à l'ensemble des réflexions sur les marques consiste à évoquer le problème de l'évaluation comme un frein majeur à leur prise en compte à l'actif distinctement du goodwill. En effet, le problème principal soulevé par la reconnaissance des marques concerne 
la possibilité de disposer d'une mesure fiable de la marque. Par ailleurs, la possibilité de disposer d'une méthode d'évaluation fiable de la marque conditionne aussi la politique de dépréciation choisie pour celle-ci. Or, lorsqu'on parle de « juste valeur », la responsabilité en est laissée aux managers (pour l'ASB et la $\mathrm{COB}$ ) ou aux préparateurs des comptes et auditeurs (IASC). Les organismes de réglementation se désengagent lorsqu'il s'agit d'évaluer une marque à sa juste valeur. Faut-il interpréter cette attitude en termes de flexibilité ou d'impuissance ?

\begin{tabular}{|c|c|c|c|c|}
\hline & ROYAUME-UNI & FRANCE & ALLEMAGNE & IASC \\
\hline $\begin{array}{l}\text { Activation d'une } \\
\text { marque acquise de } \\
\text { manière isolée }\end{array}$ & $\begin{array}{c}\text { SSAP 22 } \\
\text { oui (non explicite) } \\
\text { FRED 12/ FRS10 } \\
\text { oui } \\
\end{array}$ & $\begin{array}{c}\text { Oui } \\
\text { (PCG I 25) }\end{array}$ & $\begin{array}{c}\text { oui } \\
(\$ 248 \text { et } \$ 246 \\
\text { du HGB })\end{array}$ & oui \\
\hline $\begin{array}{l}\text { Activation d'une } \\
\text { marque provenant } \\
\text { de l'achat d'un } \\
\text { fonds de commerce }\end{array}$ & $\begin{array}{c}\text { SSAP 22 } \\
\text { oui, à leur juste valeur }\end{array}$ & oui & $\begin{array}{c}\text { oui } \\
(\mathrm{BGH})\end{array}$ & pas concerné \\
\hline $\begin{array}{l}\text { Activation d'une } \\
\text { marque acquise } \\
\text { lors de } \\
\text { regroupements }\end{array}$ & $\begin{array}{c}\text { SSAP } 22 \text { oui,à leur juste } \\
\text { valeur } \\
\text { ED } 52 \text { non, à laisser dans le } \\
\text { goodwill } \\
\text { FRED } 12 \text { / FRS } 10 \text { oui, à } \\
\text { l'aide de méthodes } \\
\text { développées par des experts }\end{array}$ & $\begin{array}{c}\text { oui (CNC, } 1990 \text { doc } \\
\text { n`85 qui vaut avis) } \\
\text { et encouragée par la } \\
\text { COB si la marque } \\
\text { est identifiable et } \\
\text { que l'on dispose } \\
\text { d'une méthode } \\
\text { d'évaluation précise }\end{array}$ & $\begin{array}{c}\text { oui } \\
\text { (§301 al. } 1 \text { HGB) }\end{array}$ & $\begin{array}{l}\text { oui, à leur juste valeur à } \\
\text { l'aide de cash-flows } \\
\text { actualisés } \\
\text { s'ils sont indépendants } \\
\text { de ceux générés par } \\
\text { d'autres actifs }\end{array}$ \\
\hline Marques créées & $\begin{array}{c}\text { ED } 52 \text { non } \\
\text { FRED } 12 \text { non, par } \\
\text { déduction car ni norme } \\
\text { spécifique ni marché actif }\end{array}$ & $\begin{array}{l}\text { oui, par déduction } \\
\text { (PCG) } \\
\text { rapport } \mathrm{n}^{\circ} 94 \mathrm{du} \\
\mathrm{CNC} \text { (qui ne vaut } \\
\text { pas avis) en faveur } \\
\text { de cette pratique }\end{array}$ & $\begin{array}{c}\text { Interdit } \\
(\S 248 \mathrm{HGB})\end{array}$ & $\begin{array}{c}\text { E 50, non } \\
\text { ce n'est pas probable } \\
\text { E 60, IAS } 38 \text { non } \\
\text { c'est interdit }\end{array}$ \\
\hline $\begin{array}{c}\text { Traitement } \\
\text { comptable des } \\
\text { marques }\end{array}$ & $\begin{array}{c}\text { ED } 52 \text { amt sur } 20 \text { ans (40 } \\
\text { ans max) } \\
\text { FRED 12/FRS10 } \\
\text { amt sur } 20 \text { ans } \\
\text { (présomption) } \\
\text { amt sur une durée sup. à } 20 \\
\text { ans ou pas du tout } \\
\text { test de dépréciation dans les } \\
\text { deux derniers cas }\end{array}$ & $\begin{array}{l}\text { amortissement non } \\
\text { prévu par le PCG et } \\
\text { non désiré par la } \\
\text { COB. Ce n'est } \\
\text { toutefois pas interdit } \\
\text { Provision pour } \\
\text { dépréciation } \\
\text { recommandée }\end{array}$ & $\begin{array}{c}\text { amortissement sur } \\
\text { une durée de } 3 \text { à } 5 \\
\text { ans }\end{array}$ & $\begin{array}{c}\text { E } 60, \text { IAS } 38 \\
\text { amortissement sur une } \\
\text { durée de } 20 \text { ans } \\
\text { (présomption) } \\
\text { ou sur une durée de vie } \\
\text { sup. avec justification } \\
\text { sans que celle-ci puisse } \\
\text { être illimitée. } \\
\text { Test de dépréciation à } \\
\text { chaque inventaire dans ce } \\
\text { cas }\end{array}$ \\
\hline $\begin{array}{l}\text { Activation du } \\
\text { goodwill créé }\end{array}$ & Interdit & Interdit & Interdit & Interdit \\
\hline $\begin{array}{l}\text { Traitement } \\
\text { comptable du } \\
\text { goodwill } \\
\text { résiduel acquis } \\
\text { dans les comptes } \\
\text { consolidés }\end{array}$ & $\begin{array}{c}\text { FRED 12/ FRS } 10 \\
\text { activation obligatoire et } \\
\text { alignement des règles avec } \\
\text { le traitement comptable des } \\
\text { marques }\end{array}$ & $\begin{array}{c}\text { activation avec } \\
\text { amortissement sans } \\
\text { durée maximale } \\
\text { fixée ou imputation } \\
\text { sur les réserves dans } \\
\text { certains cas } \\
\text { réglementés }\end{array}$ & $\begin{array}{c}\text { activation et } \\
\text { amortissement } \\
\text { selon les règles } \\
\text { relatives au fonds } \\
\text { de commerce : sur } \\
4 \text { ans ou durée de } \\
\text { vie (fiscalement } 15 \\
\text { ans) ou imputation } \\
\text { sur les réserves } \\
\end{array}$ & $\begin{array}{c}\text { IAS 22, amortissement } \\
\text { sur } 5 \text { ans avec une durée } \\
\text { inférieure à } 20 \text { ans max. } \\
\text { E 60, IAS } 38 \text { activation } \\
\text { obligatoire et alignement } \\
\text { des règles avec le } \\
\text { traitement comptable des } \\
\text { marques }\end{array}$ \\
\hline
\end{tabular}

Tableau 1- Tableau récapitulatif des normes et des projets en cours 


\subsection{Des doctrines comptables contingentes}

Les différences doctrinales peuvent être expliquées pour une bonne part par le contexte économique, juridique et fiscal dans lequel évolue chaque pays; contexte qui peut avoir une influence sur l'objectif des états financiers et par là même sur les principes fondamentaux privilégiés par les diverses réglementations. Ces principes ne sont pas sans incidence sur les règles de comptabilisation et d'évaluation des actifs et donc des marques.

Ainsi, du point de vue de l'importance des marchés financiers, le Royaume-Uni se distingue fondamentalement des deux autres pays en privilégiant le point de vue des actionnaires. Du fait du contexte dans lequel évoluent ces sociétés, on peut penser que celles-ci vont présenter des états financiers susceptibles d'offrir une information utile aux décisions des investisseurs. C'est aussi ce qui explique que le true and fair view exerce une influence plus forte au Royaume-Uni qu'en Allemagne et en France. Ces derniers ont une vision plus large de la comptabilité qui doit satisfaire tous les partenaires de l'entreprise.

Alors que les classifications des systèmes comptables ont tendance, à ce stade, à placer dans le même groupe la France et l'Allemagne, nous avons pu mettre en évidence des différences sensibles entre ces deux pays sur d'autres points, différences qui ne vont pas être sans conséquence sur la reconnaissance des marques à l'actif du bilan.

Le processus de normalisation, par exemple, semble plus souple et adapté en France qu'en Allemagne. De même, si la fiscalité a son importance en France, son influence est tout de même moins forte puisque la comptabilité ne va pas jusqu'à s'aligner sur elle. Le problème fiscal a, par ailleurs, permis de dégager l'idée selon laquelle c'est sur les comptes consolidés que devra s'exercer toute réflexion visant à harmoniser les comptes et toute tentative visant à rapprocher les pratiques nationales de celles en vigueur sur un plan international (VAN HULLE, 1995).

Parmi les principes privilégiés par les différentes doctrines, certains sont, en effet, plus proches d'une conception patrimoniale peu encline à reconnaître les marques, d'autres au 
contraire constituent des arguments en leur faveur, s'ils sont privilégiés. Ainsi l'Allemagne, dominée par le principe de prudence et celui de prépondérance accorde logiquement une large part aux coûts historiques ce qui amène à ne reconnaître les marques que lorsqu'il est possible de disposer d'un coût d'acquisition. Tandis que la France semble vouloir se dégager progressivement d'une optique patrimoniale et du principe de prudence qualifié de «mal nécessaire » (CAILLIAU, 1996) et privilégier à la pertinence. Cette évolution est, par ailleurs, cohérente avec les travaux en faveur des marques amorcés au sein du Conseil National de la Comptabilité. Quant au Royaume-Uni, la domination du principe de substance over form explique qu'il accorde aux marques une attention toute particulière au niveau doctrinal.

\subsection{Des méthodes d'évaluation disparates}

Le débat sur la mesure comptable ne saurait être complet sans une explicitation détaillée des principales méthodes en vigueur en matière d'évaluation, de manière à juger de leur adéquation, en matière de fiabilité et de pertinence, avec l'objectif d'activation de la marque.

Le problème réside justement dans la possibilité de trouver une méthode qui rassemblerait ces deux qualités à la fois. Une valeur de marché que l'on pourrait obtenir grâce à l'observation de transactions externes similaires semble alors s'imposer d'elle-même (PIERRAT, MARTORY, 1996). Il faut toutefois renoncer à une telle méthode dans le cas où les transactions portant sur des marques isolées sont peu fréquentes et rarement comparables. Il est alors nécessaire de se tourner vers d'autres méthodes.

Certaines méthodes existent qui tiennent compte de la réalité économique de la marque. Dans certains cas précis, des méthodes d'évaluation par les flux (ex : méthode des royalties et différentiel de marge) peuvent se révéler adaptées. Toutefois, leurs difficultés communes résident dans la fixation de paramètres (horizon d'actualisation, taux d'actualisation etc...) qui impliquent un certain nombre de manipulations pouvant mettre en jeu leur fiabilité. 
Les méthodes multi-critères ${ }^{8}$ sont intéressantes à de nombreux points de vue. Comme les approches par les flux elles donnent une valeur monétaire à la marque. La multitude de critères et leur nécessaire monétarisation rendent inévitable une part de subjectivité, cependant, la fixation de la plupart des paramètres se fait au terme d'une analyse approfondie de la force de la marque. Plus complètes, ces méthodes se révèlent pertinentes pour l'objectif d'activation tout en réduisant au maximum la subjectivité. Par ailleurs, ces méthodes permettent, dans le même temps, une analyse de la marque en termes de forces et faiblesses, qui peut être utile pour sa gestion et son contrôle. Par là même, elles permettent de concilier les objectifs de différents départements de l'entreprise.

D’une manière générale, on a pu constater que la pertinence va souvent de pair avec une bonne part de subjectivité. Or, si on continue à privilégier la fiabilité à la pertinence, ce qui revient à conserver uniquement la méthode du coût d'acquisition, on a peu de chances de voir progresser la reconnaissance des marques au bilan puisque cette méthode laisse de côté les marquées créées et celles acquises lors d'un regroupement.

La question qui se pose, au-delà de la validité de la méthode d'évaluation en vue de l'activation, est plutôt l'objectif poursuivi par les états financiers : une approche tournée vers le passé et déconnectée de la réalité économique qui conduit à retenir seulement des méthodes fiables mais non pertinentes ou une approche tournée vers l'avenir qui autorise l'inclusion de valeurs incertaines, lorsqu'elles sont susceptibles d'avoir un impact sur la performance de l'entreprise. Quelle que soit l'approche retenue, il importe surtout de ne pas se montrer plus exigeant envers la marque que vis à vis des actifs matériels. 


\section{Des disparités au niveau des pratiques des entreprises : effets secteurs et effets pays}

\subsection{Méthodologie : deux niveaux d'analyse}

L'analyse du cadre institutionnel a permis de dégager les points clés de la mesure comptable et d'établir un certain nombre d'hypothèses qui ont pu être regroupées autour de quatre thèmes :

- le premier thème porte sur la divulgation de l'information relative à l'existence et au montant des marques dans les comptes consolidés et dans le rapport annuel

- le deuxième thème porte sur le traitement comptable des marques ultérieurement à leur reconnaissance. On s'intéresse plus particulièrement aux pratiques en matière de divulgation de la méthodologie d'évaluation de la marque et en matière de politique de dépréciation adoptée.

- les deux derniers thèmes concernent le traitement de l'écart de première consolidation et du goodwill du fait de leur relation étroite avec la présence des marques à l'actif.

Aux diverses hypothèses formulées sur la différenciation des pratiques par pays $(\mathrm{H} 1, \mathrm{H} 4, \mathrm{H} 7$, H10, H13, H16), sont ajoutées celles portant sur la différenciation des pratiques par secteurs (H2, H5, H8, H11, H14, H17), du fait du rôle différent que peut jouer la marque suivant le secteur d'appartenance de l'entreprise ${ }^{9}$. Enfin, certaines hypothèses apparaissent comme complémentaires permettant de mettre en cohérence les informations (H3, H6, H9, H 12, H15).

L'étude empirique est constituée de deux volets et permet de recouvrir deux séries d'objectifs :

- Au niveau positif : observer les pratiques des entreprises

- Au niveau normatif : recueillir les opinions de responsables comptables et financiers

Cette double étude empirique, exploitant conjointement des informations externes disponibles à travers les plaquettes annuelles et des informations internes, recueillies à partir de questionnaires entretiens, devrait permettre de satisfaire à la nécessité de triangulation méthodologique (USUNIER et al. , 1993). 
Ier volet : Une étude statistique réalisée sur la base de l'information externe diffusée par les sociétés dans leurs plaquettes annuelles a permis de tester les hypothèses. Elle porte sur 150 groupes répartis également par pays (3 pays) et par secteurs (5 secteurs) et a été effectuée par consultation directe des plaquettes 1996. Les informations ont été traitées à l'aide du logiciel SPHYNX et analysées par des tests de Chi-deux permettant de mesurer l'indépendance entre deux variables. Une synthèse a été effectuée à travers une Analyse Factorielle des Correspondances Multiples (AFCM).

$\underline{2^{\text {ème }}}$ volet $:$ Une étude plus qualitative, effectuée sur la base de l'information interne recueillie par questionnaires/entretiens, a permis d'affiner et de préciser les résultats obtenus mais aussi d'enrichir les conclusions par les opinions des responsables comptables et financiers. Un questionnaire a été envoyé à l'ensemble des groupes étudiés et a obtenu un taux de réaction de $46 \%$ qui correspond à des réponses positives et négatives -ces dernières ont bien souvent apporté des éléments de réponse-. 22 responsables ont finalement accepté de répondre positivement au questionnaire et dans certains cas de se prêter à un entretien, soit un taux de réponse positive de $15 \%$.

\subsection{Les résultats de l'étude statistique : un effet pays prédominant}

Sur les 17 hypothèses présentées et comme l'indique le tableau récapitulatif des hypothèses présenté infra, 10 sont vérifiées tandis que 7 d'entre elles ne sont pas vérifiées. Une constante indique que l'effet pays l'emporte sur l'effet secteur. 


\section{$\underline{\text { Divulgation de l'information sur l'existence et le montant des marques }}$}

\section{comptes consolidés}

H1 Les pratiques en matière de reconnaissance des marques à l'actif diffèrent selon la nationalité de l'entreprise Vérifiée

H2 Les pratiques en matière de reconnaissance des marques à l'actif diffèrent selon le secteur d'appartenance de l'entreprise Non Vérifiée

H3 L'inscription d'une marque sur une ligne distincte à l'actif dépend de son importance par rapport aux autres immobilisations incorporelles Vérifiée

\section{rapport annuel}

H4 Les pratiques en matière d'identification des marques dans le rapport annuel diffèrent selon la nationalité de l'entreprise. Non Vérifiée

H5 Les pratiques en matière d'identification des marques dans le rapport annuel diffèrent selon le secteur d'appartenance de l'entreprise. Vérifiée

H6 L'identification des marques dans le rapport annuel implique une reconnaissance de celles-ci à l'actif. Non Vérifiée

Traitement des marques

\section{Evaluation des marques}

H7 Les pratiques en matière de divulgation des critères d'évaluation varient selon la nationalité de l'entreprise. Vérifiée

H8 Les pratiques en matière de divulgation des critères d'évaluation varient selon le secteur d'appartenance de l'entreprise. Vérifiée pour la France

H9 Les entreprises possédant des marques à l'actif divulguent aussi les détails quant à leur évaluation à l'inventaire. Non Vérifiée

\section{Mode de dépréciation des marques}

H10 Les politiques de dépréciation des marques varient suivant selon la nationalité de l'entreprise. Vérifiée

H11 Les politiques de dépréciation des marques varient suivant selon le secteur d'appartenance de l'entreprise. Non Vérifiée

H12 La divulgation des critères d'évaluation de la marque incite les entreprises à ne pas amortir les marques.

\section{Vérifiée}

\section{$\underline{\text { Traitement de l'écart de première consolidation }}$}

H13 Les pratiques en matière d'affectation de l'écart de première consolidation aux marques varient suivant la nationalité de l'entreprise. Vérifiée

H14 Les pratiques en matière d'affectation de l'écart de première consolidation aux marques varient suivant le secteur d'appartenance de l'entreprise. Non Vérifiée

H15 La divulgation des critères d'évaluation de la marque incite les entreprises à affecter l'écart de première consolidation aux marques. Vérifiée

\section{Traitement du goodwill.}

H16 Les pratiques en matière de traitement du goodwill diffèrent selon la nationalité de l'entreprise. Vérifiée

H17 Les pratiques en matière de traitement du goodwill diffèrent selon le secteur d'appartenance de l'entreprise. Non Vérifiée

Tableau 2 - Tableau récapitulatif des hypothèses 
Ainsi, les résultats indiquent que les pratiques en matière de divulgation de l'information relative aux montants des marques et aux méthodes d'évaluation dans les comptes consolidés ainsi qu'en matière de mode de dépréciation des marques sont différenciées de façon très significative par pays et peu, voire pas du tout, par secteurs. Ce résultat est aussi valable pour le traitement du goodwill et de l'écart de première consolidation.

En revanche, les pratiques en matière d'identification des marques dans le rapport annuel sont très différenciées par secteurs et non par pays. Toutefois, l'identification des marques n'implique pas forcément une inscription de celles-ci à l'actif. On apprend, par ailleurs que lorsque les marques figurent à l'actif, celles-ci représentent près de $80 \%$ des immobilisations incorporelles.

On apprend aussi que les entreprises qui inscrivent des marques à l'actif ne divulguent pas pour autant les détails relatifs à la méthode d'évaluation employée. Toutefois, les entreprises qui divulguent de tels détails sont aussi celles qui ont tendance à affecter l'écart de première consolidation aux marques et à ne pas les amortir.

L'Analyse Factorielle des Composantes Multiples a permis, ensuite, de synthétiser et de confirmer les résultats obtenus en identifiant trois pôles d'entreprises comme l'indique la figure suivante. Chaque pôle comporte une nationalité qui est sur représentée.

Ces résultats indiquent que les groupes britanniques sont généralement hostiles à l'activation des marques et s'opposent alors aux entreprises françaises en faveur de leur présence à l'actif du bilan, tandis que les entreprises allemandes se distinguent par leur position imprécise et prudente. Toutefois, même si les firmes britanniques font largement partie du deuxième pôle identifié, certaines sont à ranger dans le pôle 1 avec les entreprises françaises. En effet, lorsque les entreprises britanniques ont des immobilisations incorporelles à l'actif, il s'agit presque exclusivement de marques jamais amorties, ces entreprises ont alors une attitude vis-à-vis des marques similaire à celle des entreprises françaises. 


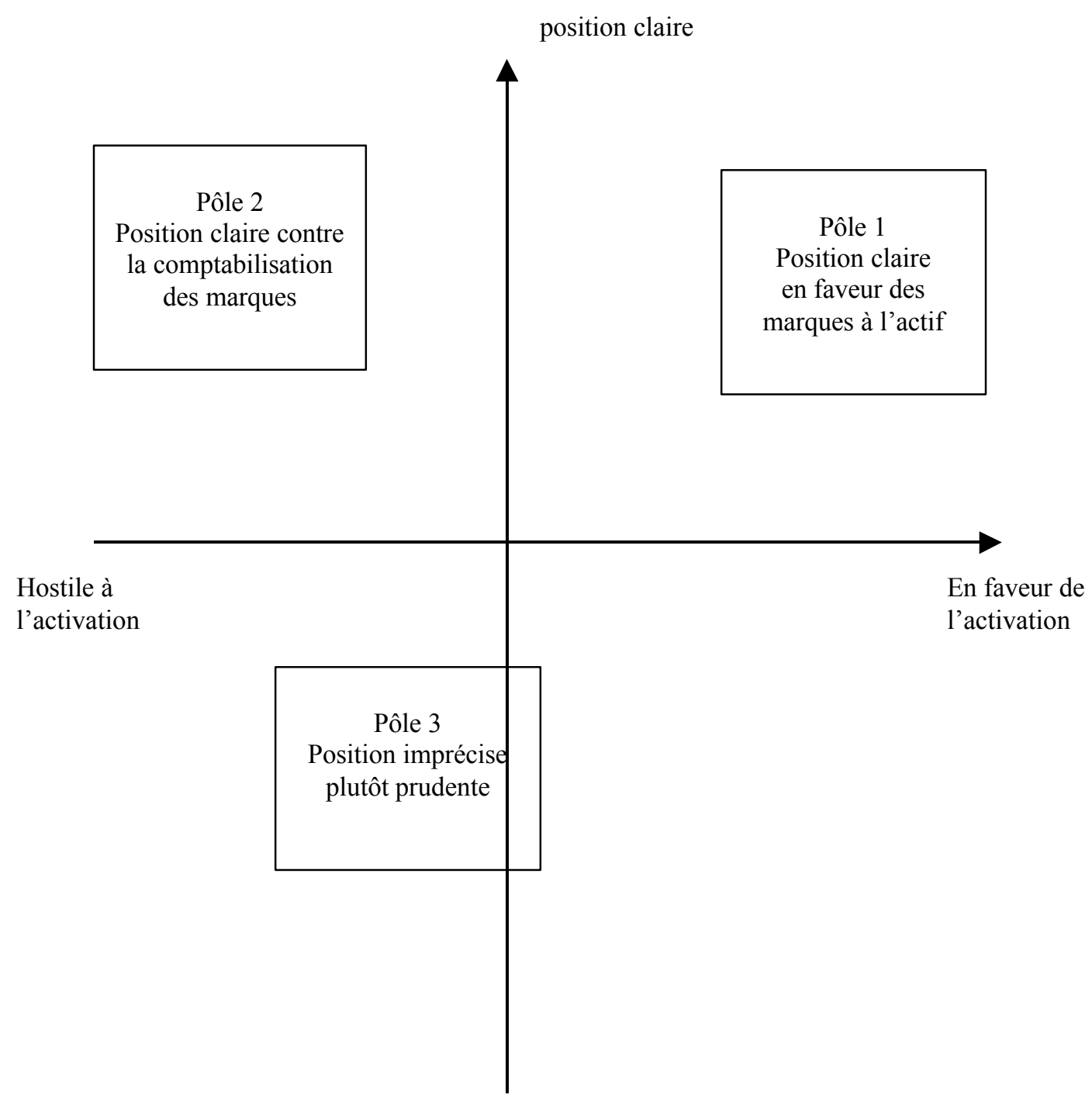

Figure 1- les trois pôles d'entreprises

Position imprécise

1er pôle : position clairement en faveur des marques

- sur représentation des entreprises françaises

- immobilisations incorporelles à l'actif

- marques à l'actif sur une ligne distincte à l'actif ou dans l'annexe

- écart de $1^{\text {ère }}$ consolidation affecté aux marques

- marques non amorties

2ème pôle : position clairement hostile à l'activation des marques

- sur représentation des entreprises britanniques

- aucune information sur les immobilisations incorporelles

- pas de marques à l'actif

3ème pôle : position imprécise et prudente

- sur représentation des entreprises allemandes

- immobilisations incorporelles à l'actif englobées avec le goodwill

- marques à l'actif sans certitude

- marques amorties 


\subsection{Les résultats aux questionnaires et entretiens : l'élargissement du débat}

Les réponses fournies par les questionnaires/entretiens sont dans l'ensemble cohérentes avec les pratiques des entreprises telles qu'elles ressortent dans les plaquettes. Les opinions des responsables comptables et financiers ont, par ailleurs, permis d'enrichir les conclusions en apportant un éclairage différent au problème de la mesure comptable des marques.

Il en ressort trois points essentiels. Une harmonisation au niveau international est souhaitée par la majorité des entreprises, les questions qui semblent encore poser problème concernent l'amortissement des marques et leur évaluation. Plusieurs responsables français et britanniques se montrent farouchement opposés à l'amortissement des marques invoquant que ces dernières ne se déprécient pas automatiquement avec le temps. Quant au problème de leur évaluation, les responsables ont souligné qu'il n'est pas possible de normaliser sur ce point. Les méthodes doivent pouvoir s'adapter aux particularités des marques et des secteurs.

Toujours en ce qui concerne l'évaluation de la marque, elle ne constitue pas un frein majeur à la reconnaissance des marques à l'actif, si ce n'est à cause d'un manque de connaissance des méthodes. On a pu observer, en effet, un décalage entre la typologie établie à partir d'articles spécialisés, et les méthodes connues par les entreprises. Cette hypothèse est confirmée par le fait que, pour les entreprises qui font appel à des organismes externes d'évaluation des marques, la prise en compte des marques ne pose plus aucun problème.

Un dernier point essentiel concerne le caractère sensible de l'information véhiculée par la valeur des marques. C'est une information confidentielle jugée stratégique. Derrière la valeur des marques et notamment des marques créées il y a montants des dépenses de communication qu'on n'est pas prêt de divulguer aux concurrents. 


\section{Conclusion et synthèse}

Si on confronte les résultats de l'étude empirique avec les conclusions obtenues par l'analyse du cadre institutionnel, on aboutit à des résultats différenciés suivant les pays. En France, les pratiques sont cohérentes avec l'intérêt manifesté pour les marques par les instances françaises de normalisation ou de réglementation. Soulignons au passage la place remarquable qu'occupe la France, tant au niveau d'avancées sur le débat doctrinal qu'en termes de pratiques de divulgation de l'information financière. En Allemagne, on observe une prudence dans la pratique qui va de pair avec la doctrine générale et le peu d'intérêt à l'égard du sujet que semble manifester la réglementation allemande. C'est au Royaume-Uni qu'on observe les résultats les plus contrastés. Il existe une divergence entre l'intérêt manifesté par les doctrines et celui exprimé par les entreprises britanniques. L'engouement pour les marques n'est le fait que d'une minorité d'entreprises qui, sur ce point, ont une position comparable à celle des entreprises françaises. Sur le problème de la mesure comptable des marques, la traditionnelle opposition entre le Royaume-Uni, d'un côté, et la France et l'Allemagne de l'autre n'est donc pas vérifiée

Certes, ces résultats ne sont valables que par rapport à un échantillon bien défini et en relation avec un certain nombre de restrictions qui ont été définies au départ. En conséquence, ils ne doivent être considérés que comme des étapes appelant à un approfondissement et à un élargissement ultérieur de la recherche. En particulier, il s'agira de se reposer le problème de l'utilité de l'information en opérant une segmentation plus fine des utilisateurs de l'information financière. De même, il faudrait pouvoir appliquer l'étude à d'autres types d'entreprises et à d'autres pays pour être en mesure de généraliser des résultats. D'autres points ont été abordés mais nécessiteraient d'être approfondis. Ainsi il serait utile, en matière de méthodes d'évaluation, d'affiner les analyses en distinguant différents types de marques et notamment les marques industrielles et les marques de grande consommation. 
Il est cependant possible de présenter une première synthèse du débat.

1) Tout d'abord, on peut se demander si la raison pour laquelle aucune norme internationale n'est opérationnelle sur ce point n'est pas le fait de fortes divergences politiques. On a pu constater que certaines entreprises se montraient farouchement opposées sur certains points (comme l'amortissement des marques) dont la résolution conditionne l'acceptation de la norme tout entière. Le problème technique de la mesure comptable fait alors place à un problème d'ordre politique.

2) Par ailleurs, il semble que le problème de l'évaluation de la marque, posée comme frein à la reconnaissance des marques semble bien souvent un prétexte émis par les réglementations. On a pu constater au niveau théorique et des pratiques que l'évaluation de la marque n'est pas insurmontable. Il n'est pas possible de réglementer sur ce point en imposant une seule méthode applicable à toutes les entreprises, ce qui ne signifie pas pour autant que l'on ne sache pas évaluer. La difficulté de l'évaluation des marques constituerait un moyen commode pour les divers organismes de réglementations de se retrancher derrière un problème technique faute de ne pas avoir su trouver un accord satisfaisant l'ensemble des parties. Cette conclusion est confirmée par le fait que la plupart des textes réduisent la reconnaissance des marques à une condition d'évaluation sans l'expliciter.

3) Au niveau européen, les pays étudient les questions chacun de leur côté sans qu'une véritable concertation existe, ce qui donne lieu, nous l'avons vu, à des conclusions très différentes. Le fait que l'effet pays l'emporte sur l'effet secteur pose le problème de l'harmonisation européenne dans toute son acuité. Il légitime le souci de rapprocher les cadres institutionnels et les pratiques des acteurs dans l'Europe de demain. Sous peine d'une refonte totale des normes nationales, l'harmonisation pourrait, dans un premier temps, seulement porter sur les comptes consolidés, lesquels ne participent pas à l'élaboration du revenu imposable. Il semblerait toutefois, qu'il y ait une volonté de s'aligner sur des normes internationales, comme 
le prouve l'actuelle norme de l'ASB dont les modifications apportées tiennent compte de la position en vigueur sur un plan international. Ces normes constitueraient alors peut-être la solution pour harmoniser les pratiques. L'application de la norme IAS 38 en juin 1999 introduira une nouvelle donne, modifiera les comportements. Cette dynamique entre la norme et les pratiques pourra faire l'objet d'une nouvelle analyse. 
${ }^{1}$ C'est ainsi que le CGP publie un rapport dans lequel il associe étroitement la croissance industrielle et l'investissement immatériel.

${ }^{2}$ Le rapport BARWISE et al. (1989) fait figure d'exception. Il examine, toutefois, l'utilité de l'information relative aux marques au bilan plutôt par rapport aux marchés financiers et aux banquiers

${ }^{3}$ De nombreux travaux et projets de normes ont vu le jour vers la fin des années 80 et ont connu un aboutissement à la fin des années 90

${ }^{4} \mathrm{Du}$ fait des pratiques innovantes de certaines entreprises qui ont activé des marques acquises lors de regroupements et même développées en interne. Pour plus de détails consulter ALLEN, 1990

${ }^{5}$ Seules des reflexions sur les conditions d'application d'une comptabilité d'après les règles américaines et internationales existent (GLAUM, MANDLER, 1996). Elles prennent en compte parfois l'immatériel (Von KEITZ, 1997) sans pour autant considérer le cas de la marque en tant que capital-marque. Il faut dire que cette dernière n'est séparable de l'entreprise que depuis 1992.

${ }^{6}$ La conclusion du texte E60 est d'ailleurs plus catégorique que le texte précédent E 50 puisque de «peu de chances - highly unlikely» de figurer à l'actif, on est passé à «aucune chances - will not qualify for recognition as an intangible asset ».

${ }^{7}$ Soulignons à ce sujet, qu'en rendant obligatoire l'amortissement du goodwill, l'ASB a tranché dans sa norme sur les actifs immatériels contre la méthode d'imputation sur les réserves, privilégiée par le passé au Royaume-Uni.

${ }^{8}$ Parmi les méthodes «multi-critères » les plus citées par les articles spécialisés, on pourra noter la méthode d'INTERBRAND pour le Royaume-Uni, celle de SORGEM pour la France et celle de A.C. NIELSEN pour l'Allemagne.

${ }^{9}$ On a procédé à un regroupement de plusieurs secteurs de manière à faire ressortir la signification de chacun d'eux par rapport à la marque.

S1 : Energie/ Produits de base/ Construction

S2 : Biens d'équipement/ Automobile

S3 : Autres biens de consommation

S4 : Industrie Agro-alimentaire

S5 : Distribution/ Autres services 


\section{Bibliographie}

AAKER David (1994), Le management du capital-marque, Dalloz

Accounting Standards Board (1993), Goodwill and intangibles assets, discussion paper, London

Accounting Standards Board (1997), FRS 10, Goodwill and Intangible assets, december

ALLEN David (1990), Creating value, the financial management of brands, The Chartered Institute of Management Accountants, London

BARWISE Patrick et al. (1989), Accounting for brands, Institute of Chartered Accountants of England and Wales in conjunction with London Business School, London

BARWISE Patrick (1993), Brand equity : snark or boojum, International Journal of Research in Marketing, vol. 10, $\mathrm{n}^{\circ}$ 1, March, pp. 93-104

CAILLIAU Jean-Claude (1996), Cadre conceptuel de la comptabilité en tant que que système de représentation publique de la situation économique (et financière) de l'entreprise, Revue Française de Comptabilité, n²78, Mai, pp 17-51

CASTA Jean-François (1997), La politique comptable des entreprises, Encyclopédie de gestion, tome 2, Economica, pp. 2276-2301

COLASSE Bernard (1996), La comptabilité : un savoir d'action en quête de théories, in J.M. BARBIER, Savoirs théoriques et savoirs d'action, PUF, pp. 73-89

Commissariat Général au Plan (1982), Investissement non matériel et croissance industrielle, Préparation du IXème plan 1984-1988, Rapport du groupe de travail, La documentation française

Conseil National de la Comptabilité (1990), Avis relatif au traitement comptable de l'écart $d^{\prime}$ acquisition, document $\mathrm{n}^{\circ} 85,15$ janvier

Conseil National de la Comptabilité (1992), Les marques, un actif pour l'entreprise?, Rapport de synthèse sur la comptabilisation et l'évaluation des marques développées de manière interne, doc $n^{\circ} 94$, Ministère de l'Economie et des finances

Conseil National de la Comptabilité (1997), Rapport sur les travaux de la Commission des Investissements Immatériels, document de travail interne, janvier

GLAUM Martin, MANDLER Udo (1996), Rechnungslegung auf globalen Kapitalmärkten, Gabler Verlag, Wiesbaden

HAMMANN Peter, GATHEN Andreas von der (1994), Bilanzierung des Markenwertes und kapitalmarktorientierte Markenbewertungsverfahren, Markenartikel, 5/, pp. 204-211

HOARAU Christian, TERNISIEN Michel (1997), Mesure, évaluation et analyse de l'efficacité des facteurs immatériels, Rapport pour le Commissariat Général au Plan, vol. 1, mai 
HULLE Van Karel (1995), Importance des comptes consolidés dans le cadre de l'harmonisation des règles comptables au sein de l'Union Européenne, Revue de droit comptable, ${ }^{\circ}$ 95-1, Mars, pp 41-50

International Accounting Standards Committee (1998), IAS 38, Intangible Assets, september

International Accounting Standards Committee (1997), Exposure Draft E60, Intangible assets, August

International Accounting Standards Committee (1995), Exposure Draft E50, Intangible asssets, June

KEITZ Isabel Von (1997), Immaterielle Güter in der internationalen Rechnungslegung, IDW Verlag Düsseldorf

PIERRAT Christian, MARTORY Bernard (1996), La gestion de l'immatériel, Editions Nathan

USUNIER Jean-Claude, EASTERBY-SMITH Mark, THORPE Richard (1993), Introduction à la recherche en gestion, Editions Economica

WALLISER Elisabeth (1998), La mesure comptable des marques : une étude comparée en Europe, Thèse de doctorat en Sciences de Gestion, Université Paris XII Val de Marne, ESA IRG, décembre, 484 pages 